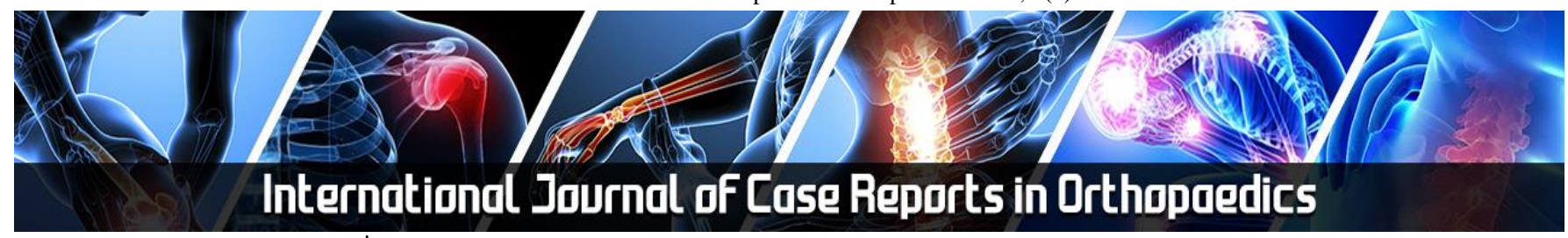

E-ISSN: 2707-8353 P-ISSN: 2707-8345 IJCRO 2021; 3(1): 18-20

Received: 16-11-2020 Accepted: 18-12-2020

\section{Ana Ribau}

Department of Orthopedics, Centro Hospitalar do Porto, Porto, Portugal

\section{Sara Elisa Diniz}

Department of Orthopedics, Centro Hospitalar do Porto, Porto, Portugal

\section{João Vale}

Department of Orthopedics, Centro Hospitalar do Porto, Porto, Portugal

José Costa e Castro

Department of Orthopedics, Centro Hospitalar do Porto, Porto, Portugal
Corresponding Author: Ana Ribau

Department of Orthopedics, Centro Hospitalar do Porto, Porto, Portugal

\section{Unicompartmental knee arthroplasty failure with tibial component migration: Case report}

\author{
Ana Ribau, Sara Elisa Diniz, João Vale and José Costa e Castro
}

DOI: https://doi.org/10.22271/27078345.2021.v3.i1a.43

\section{Abstract}

The unicompartmental knee arthroplasty (UKA) has demonstrated good medium to long-term results. However, conversion of UKA to total Knee arthroplasty (TKA) results in poorer outcomes than primary TKA. We report a case of a 59 years-old woman with a UKA failure, with tibial component migration to the popliteal fossa. A knee posterior approach was performed to extract this component and a parapatellar approach for conversion to TKA with a tibial component with sleeve and stem. At 6 months follow-up the patient has no pain, no inflammatory signs, functional range of motion $\left(5-100^{\circ}\right)$ and the radiographic study show no complication. This case highlights the importance of a celerity revision, since time can lead to loss of bone stock and component migration, making the surgery more demanding.

Keywords: unicompartmental knee arthroplasty, failure, revision, knee posterior approach

\section{Introduction}

The medial unicompartmental arthroplasty (UKA) has demonstrated good medium to longterm results ${ }^{[1,2]}$. The success of this technique begins with proper patient selection. Thus, UKA should only be considered in patients with isolated moderate-severe medial compartment osteoarthritis, correctable deformity, no ligament lesion, good range of motion and no inflammatory arthropathy ${ }^{[3]}$.

The rate of failure for UKA is like the rate of failure for primary total knee arthroplasty (TKA) ${ }^{[4]}$. Nevertheless, a meta-analysis from 2018 showed that conversion of UKA to TKA results in poorer outcomes than primary TKA. Despite that, UKA conversion and primary TKA have equivalents length of hospital stay, complications, and revision rates ${ }^{[5]}$.

\section{Case}

A 59 years-old female presented to the outpatient clinic with medial knee pain. No relevant co-morbidities were known. The imagological study showed a moderate medial compartment osteoarthritis with no ligament lesions. A cemented medial UKA (U-KneeTec - Tornier®) was performed in November 2015. No postoperative complications were reported. In April 2017, due to consecutively missing medical appointments, the patient lost follow-up. By the time, she was free of pain, had no functional limitation and the wound was completely healed without inflammatory signs or sinus tract.

In 2018, the patient was diagnosed epilepsy, but she also lost follow-up, do to missing medical appointments.

In November 2019, 4 year after the primary surgery, she arrived at the emergency department complaining of progressive knee pain. The knee had no inflammatory signs. The radiologic study showed failure of UKA with loosening of the tibial component. (figure 1)
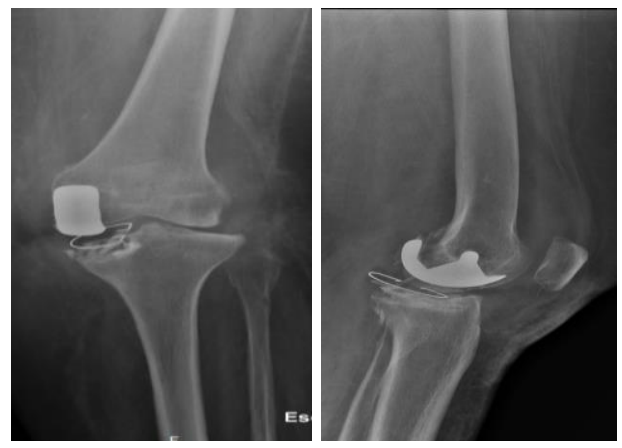

Fig 1: November $2018 \mathrm{x}$-ray showing UKA failure 
She was referred to the outpatient clinic but, once again, the patient missed the appointment. After 8 months, on 5 June 2020, she returned, complaining of knee pain, functional limitation and walking with crutches. The radiologic study showed a catastrophic mechanical UKA failure with the tibial component lost in the popliteal fossa. (figure 2)
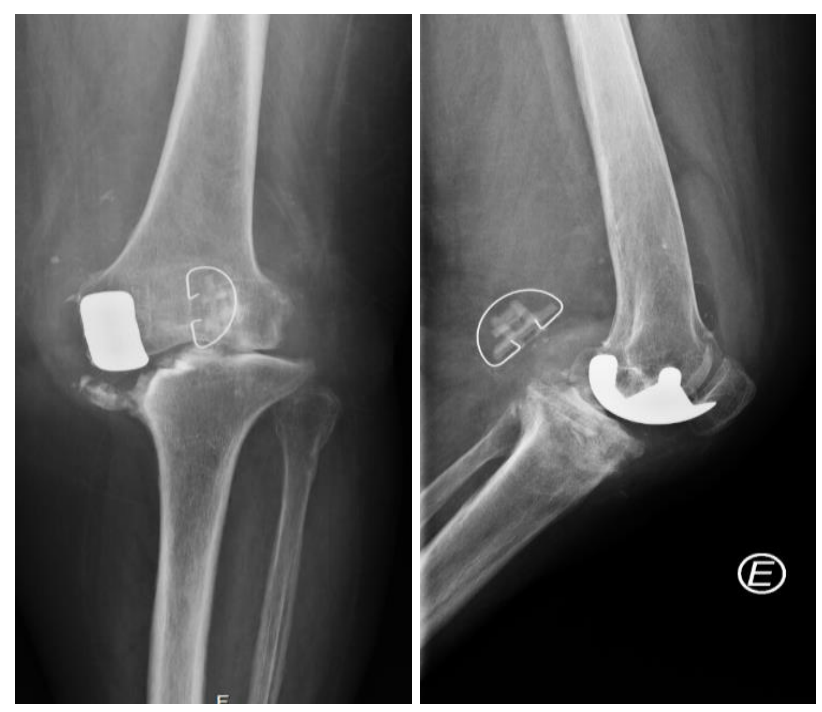

Fig 2: 5 June 2020 x-ray showing UKA failure with bone stock loss

The patient was indicated for revision. A tomographic was performed to evaluate the bone loss and the exact position of the tibial component in relation to the neurovascular bundle. The risks and benefits of the procedure were discussed, and informed consent was obtained.

She accepted the surgery. On 12 June 2020, after the preanesthetic evaluation, the patient was operated. A knee posterior approach was used to extract the lost tibial component, since it was just near the neurovascular structures. After, the previous medial parapatellar incision was used and extended $4 \mathrm{~cm}$ for the femoral component removal and conversion to TKA with a primary femoral component and a tibial revision component with sleeve and stem. Four tissue samples and the prothesis were sent to microbiology. (figure 3)
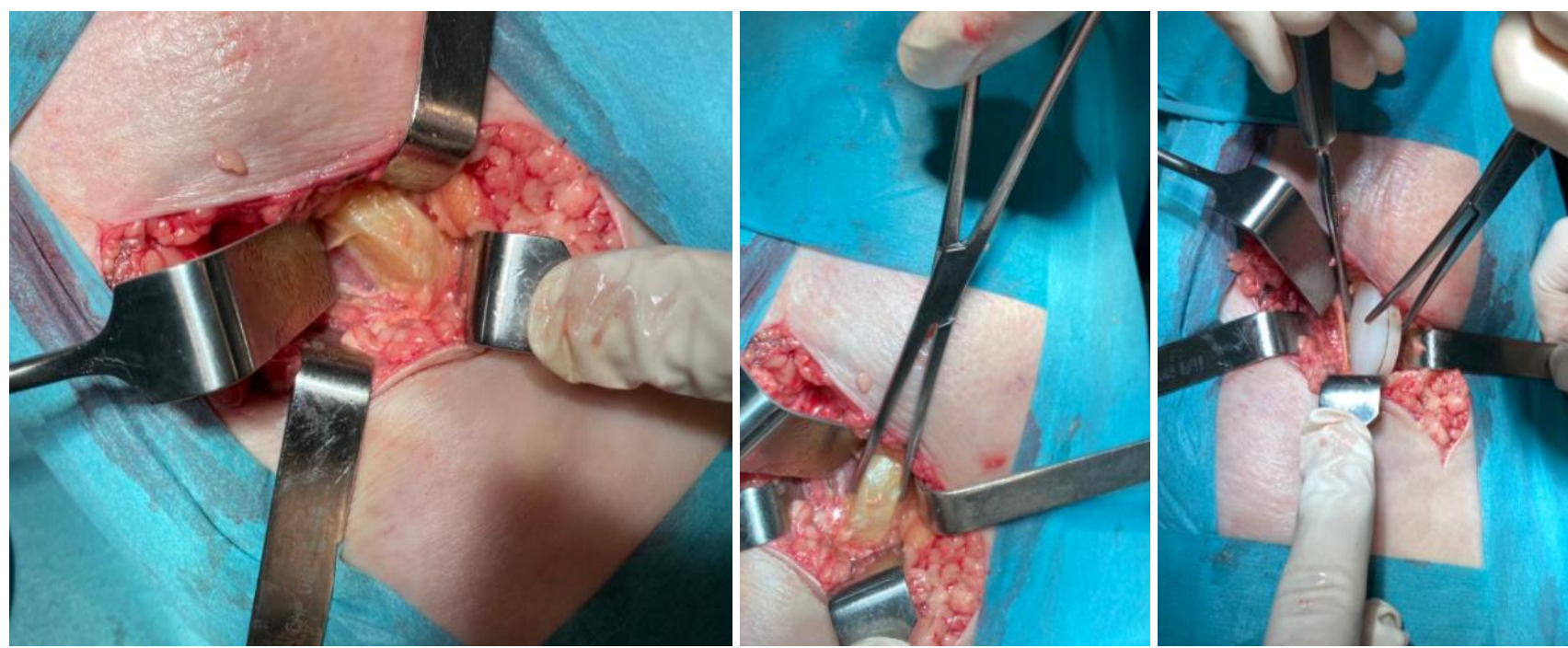

Fig 3: Intraoperative images showing the posterior approach

The surgery went without any complication and the microbiology showed all samples were sterile. The wound healed without any problem. At 6 months follow-up the patient has no pain, no inflammatory signs, functional range of motion $\left(5-100^{\circ}\right)$ and the radiographic study show no complication. (figure 4) 

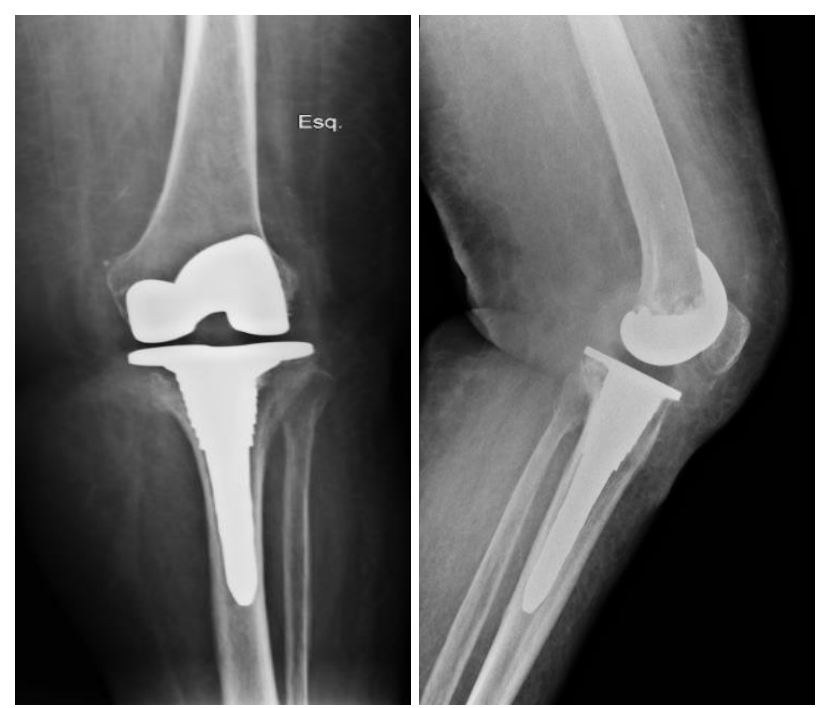

Fig 4: 6 months follow-up x-ray

\section{Discussion}

Aseptic loosening is one of the most common causes of early failure for UKA ${ }^{[6,7]}$.

The readiness identification of failure influences the complexity of the revision ${ }^{[8]}$. Some failures can be managed with a simple conservation to TKA, meaning a less invasive revision, while others leads to bone stock loss and a revision TKA with augments, stems, or bone grafts may be required [3]. Revision in a late stage of failure might limiting the clinical outcome ${ }^{[9]}$. In this case, the patient only sought care when the pain was unbearable and the gait was limited. Thereby, the revision was only possible in a late stage, without the possibility of conversion to primary TKA.

This case illustrates the importance of follow-up for the quick identification of failure and emphasizes the increase complexity that delaying revision brings.

The complexity of the surgery was increased by the position of the tibial component. A posterior knee approach can be challenging and is less common, due to the risk of neurovascular injury - including the tibial nerve, popliteal artery and vein, and common peroneal nerve.(10) Nevertheless, in this case, after the appropriate planning, it was a good option, allowing the direct visualization of the component and an easy extraction, that the authors believe would be more complex through the parapatellar approach.

\section{Conclusion}

This case warns us for the importance of follow up, since even with pain, patients may not seek health care. On the other hand, it highlights the importance of a celerity revision, empathizing the fact that a late conversion can require components with sleeves and stems.

The case demonstrates the utility and safety of a knee posterior approach in a case of UKA failure, to extract the migrated component.

\section{Conflicts of interest statement}

The author declare that there is no conflict of interests.

\section{References}

1. Johal S, Nakano N, Baxter M, Hujazi I, Pandit H, Khanduja V. Unicompartmental Knee Arthroplasty: The Past, Current Controversies, and Future Perspectives. J Knee Surg 2018;31(10):992-8.
2. Guo WS. [Role and controversy of unicompartmental knee arthroplasty in treatment of knee osteoarthritis]. Zhonghua Wai Ke Za Zhi 2020;58(6):411-5.

3. Crawford DA, Berend KR, Lombardi AV. Management of the Failed Medial Unicompartmental Knee Arthroplasty. J Am Acad Orthop Surg 2018;26(20):e426-e33.

4. Lombardi AV, Jr., Kolich MT, Berend KR, Morris MJ, Crawford DA, Adams JB. Revision of Unicompartmental Knee Arthroplasty to Total Knee Arthroplasty: Is It as Good as a Primary Result? J Arthroplasty 2018;33(7S):S105-S8.

5. Sun X, Su Z. A meta-analysis of unicompartmental knee arthroplasty revised to total knee arthroplasty versus primary total knee arthroplasty. J Orthop Surg Res 2018;13(1):158.

6. Van der List JP, Zuiderbaan HA, Pearle AD. Why Do Medial Unicompartmental Knee Arthroplasties Fail Today? J Arthroplasty 2016;31(5):1016-21.

7. Hutt JR, Farhadnia P, Masse V, LaVigne M, Vendittoli PA. A randomised trial of all-polyethylene and metalbacked tibial components in unicompartmental arthroplasty of the knee. Bone Joint J 2015;97$\mathrm{B}(6): 786-92$.

8. Berend KR, George J, Lombardi AV, Jr. Unicompartmental knee arthroplasty to total knee arthroplasty conversion: assuring a primary outcome. Orthopedics 2009, 32(9).

9. Thienpont E. Conversion of a unicompartmental knee arthroplasty to a total knee arthroplasty: can we achieve a primary result? Bone Joint J 2017;99-B(1A):65-9.

10. Faucett SC, Gannon J, Chahla J, Ferrari MB, LaPrade RF. Posterior Surgical Approach to the Knee. Arthrosc Tech 2017;6(2):e391-e5. 